\title{
The Theoretical Prediction of Thermophysical properties, HOMO, LUMO, QSAR and Biological Indics of Cannabinoids (CBD) and Tetrahhdrocannabinol (THC) by Computational Chemistry
}

\author{
Ajoy Kumer ${ }^{(D)}$ a,*, Nuruzzaman Sarkar ${ }^{b}$, Sunanda Paul c, Afroza Zannat ${ }^{a}$
}

\begin{tabular}{|c|c|}
\hline ARTICLE INFO & A B S T RACT \\
\hline $\begin{array}{l}\text { K E Y W O R D S } \\
\text { HyperChem } 8.0 .10 \\
\text { Cannabinoid } \\
\text { Tetrahhdrocannabinol } \\
\text { QSAR } \\
\text { Thermodynamic parameters } \\
\text { HOMO-LUMO } \\
\text { NMR }\end{array}$ & $\begin{array}{l}\text { Some thermophysical parameters of CBD and THC such as free } \\
\text { energy, entropy, dipole moment, binding energy, nuclear energy, } \\
\text { electronics energy, heat of formation, and chemical reactivity like } \\
\text { HOMO (Occupied Molecular Orbital Highest) and LUMO (Lowest } \\
\text { Unoccupied Molecular Orbital, HOMO-LUMO gap, ionization } \\
\text { potential and electron affinity were calculated via semi-empirical } \\
\text { and molecular mechanic method. For the characterization, the IR } \\
\text { vibration spectroscopy, NMR in case of coupling and shielding } \\
\text { constant were calculated. The Quantitative Structure Activity } \\
\text { Relation (QSAR) properties of molecules like charge density, surface } \\
\text { area grid, volume, LogP, polarizability, refractivity, molecular mass } \\
\text { were determined using the HyperChem } 8.0 .10 \text { program. Using the } \\
\text { thermophysical and QSAR data, the IC } \mathrm{I}_{50} \text { and pHIC } \text { D }_{50}\left(-\operatorname{logIC}_{50}\right) \text { was } \\
\text { developed which is referred as biological activity parameter. }\end{array}$ \\
\hline
\end{tabular}

G RA P H I C A L A B S T RACT

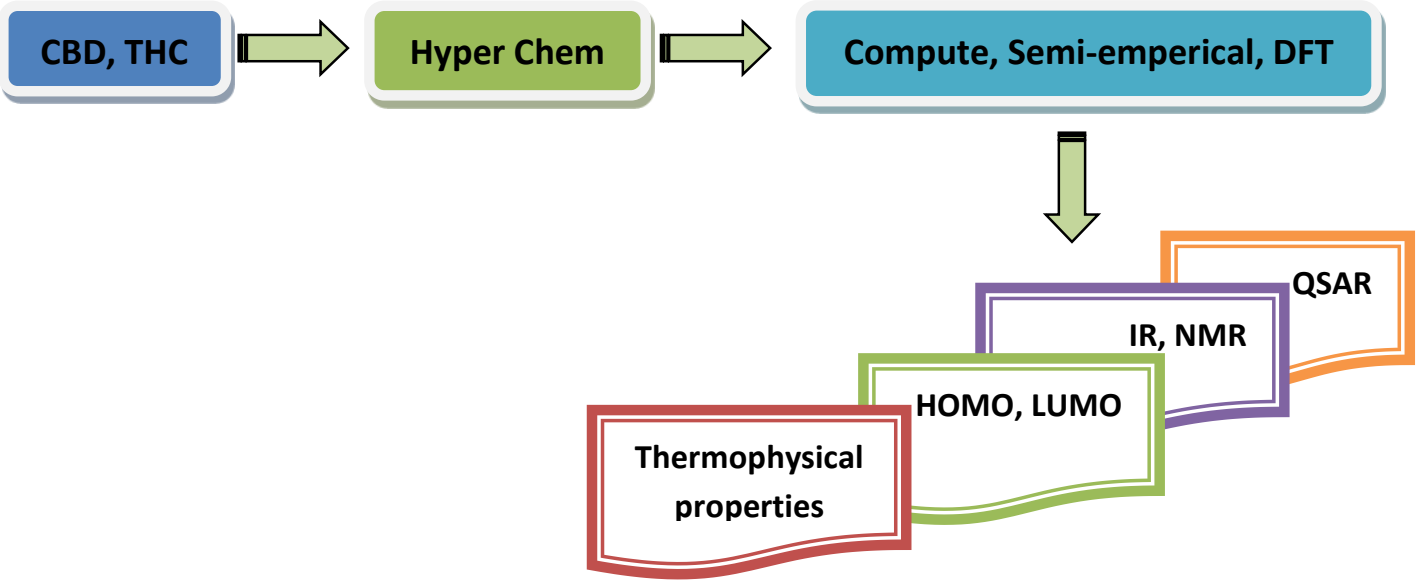

* Corresponding author's E-mail address: kumarajoy.cu@gmail.com, Tel.: +881770568699

a Department of Chemistry, European University of Bangladesh, Dhaka-1216, Bangladesh

b Department of Physics, European University of Bangladesh, Dhaka-1216, Bangladesh

c Department of Biochemistry and Molecular Biology, University of Chittagong, Chittagong,Hathazari-4334,

Bangladesh 


\section{Introduction}

Cannabinoids, a type of compounds unique to the cannabis sativa plant, are known to exhibit various physical and psychological effects upon expenditure of marijuana [1]. In the recent time, more than 100 natural cannabinoids was identified from Cannabis Sativa plant [2], at which (-)-trans- $\Delta^{9}$ - tetrahydrocannabinol (THC) and can nabidiol (CBD) are considered the two most pharmacologically active molecule in the area of drug discovery $[3,4]$. In the case of morphine based drug for pain killer was to be failed but CBD and THC based drug are to be highly active for the treatment different diseases for both of adult and young in each of time morning or other time [5-7]. The physiochemical and pharmaceutical activity of CBD and THC is completely opposite to each other on Human Brain Function and Psychopathology [8]. THC, the main psychoactive component of marijuana attributed with the euphoric effect, is also reportedly responsible for the stimulation of appetite and reduction of pain as well as increased anxiety, paranoia and impairment of memory, among many other effects $[5,9,10]$. Despite of the potential therapeutic interest of these naturally occurring CBD derivatives, only a few related pharmacological studies have been reported on the THC and CBD. Almost all the previous study intense to develop the THC and CBD for the drug discovery where these were established the properties of nonsteroidal anti-inflammatory noble drug [11 13].

The other most important implement of CBD and THC are to use in the food and beverage products which was introduced in the United States in 2017 [14, 15]. In the same ways, it is to use in energy drinks and protein bar switch acting as vitamin or herbal additives. The vital role of THC and CBD play in human body in blood transportation by lipoproteins and albumin and chemically characterized as hydrophobic nature which is responsible for toxicity in case of drug use $[16,17]$. The hydrophobic compounds may be transported from the cell membrane through the aqueous milieu by soluble intracellular carriers [18]. In this case, the computational chemistry was used to estimate the value of $\log P$ from the calculation of QSAR study and which negative value indicates the hydrophobicity and positive value indicates the hydrophilicity. The both of hydrophilicity and hydrophobicity play an important role in biochemical interactions and bioactivity with protein which may be explain in the term of binding energy. On the other the chemical reactivity of CBD and THC can be measured in term of HOMO and LUMO computing by MP3 method. 


\section{Computing method}

HyperChem 8.0.1 is a path of molecular modeling program which permits to build and analyze different molecular structures and to determine their physicochemical and thermophysical properties. In order to create the spatial chemical structure of each calculated molecule, the two-dimensional structure of the molecule shall be built stepby-step by drawing. Then hydrogen atoms are automatically added from building option and doing model building and chemical structure is converted into 3D structure. The first step in getting the main characteristic parameters of molecules is to optimize the molecular structure to obtain a minimum free energy. This is usually done using the algorithm Polak-Ribiere with maximum gradient set at $0.001 \mathrm{kcal} /(\mathrm{mol})$. The PM3 method is derived from Parametric Method number 3 from computational chemistry and included in the semiempirical method for the quantum calculation of molecular structure. PM3 was used the Hamiltonian and it is parameterized to reproduce a large number of molecular properties [19].

After optimization is achieved, the theoretical physical properties are calculated such as-as free energy, entropy, dipole moment, binding energy, nuclear energy, electronics energy, heat of formation, the energy of frontier orbital, HOMO (Occupied Molecular Orbital Highest) and LUMO (Lowest Unoccupied Molecular Orbital), and QSAR properties of molecules like charge density, surface area grid, volume, LogP, polarizability, refractivity, molecular mass, were calculated using the by QSAR.

\section{Result and Discussion}

\section{Structure optimized}

A representation of the molecular structural optimization which contains the values of the reactivity indices is called the reactive molecular diagram. The optimized structures of molecules using the HyperChem 8.0.10 software are provided in Figure 1 . The molecular symmetry is a very powerful tool established the reactivity computing on the basis of HyperChem. Using the molecular optimization, it can be found that the both of molecules containing more than one symmetry axis. The molecules involves to the class asymmetry, and nonplanar and they have more than one element of symmetry and the plane of the molecule.

\section{Bond length}

In the simple way for length of the bond is explained between two atoms involving two nucleus approximately the sum of the covalent radii of the two atoms [20]. For covalent bonds, bond energies and bond lengths depend on many factors like 
electron affinities, sizes of atoms, differences in their electro-negativity, and the overall structure of the molecule. There is a general trend in that the shorter the bond length, the higher the bond energy. Similar bond length

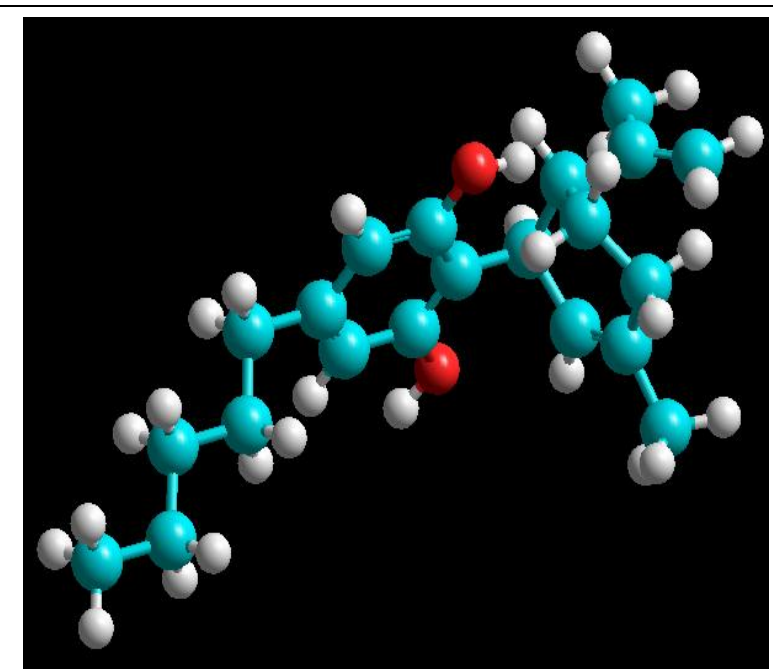

Ball and cylinder shape: Cannabidiol (CBD) indicates the similarity and molecular symmetry. In the both molecules the bond length in the carbon carbon, carbon hydrogen and carbon oxygen are 1.46, 1.09, and 0.96 .

Figure 1. Optimized Structure of CBD and THC
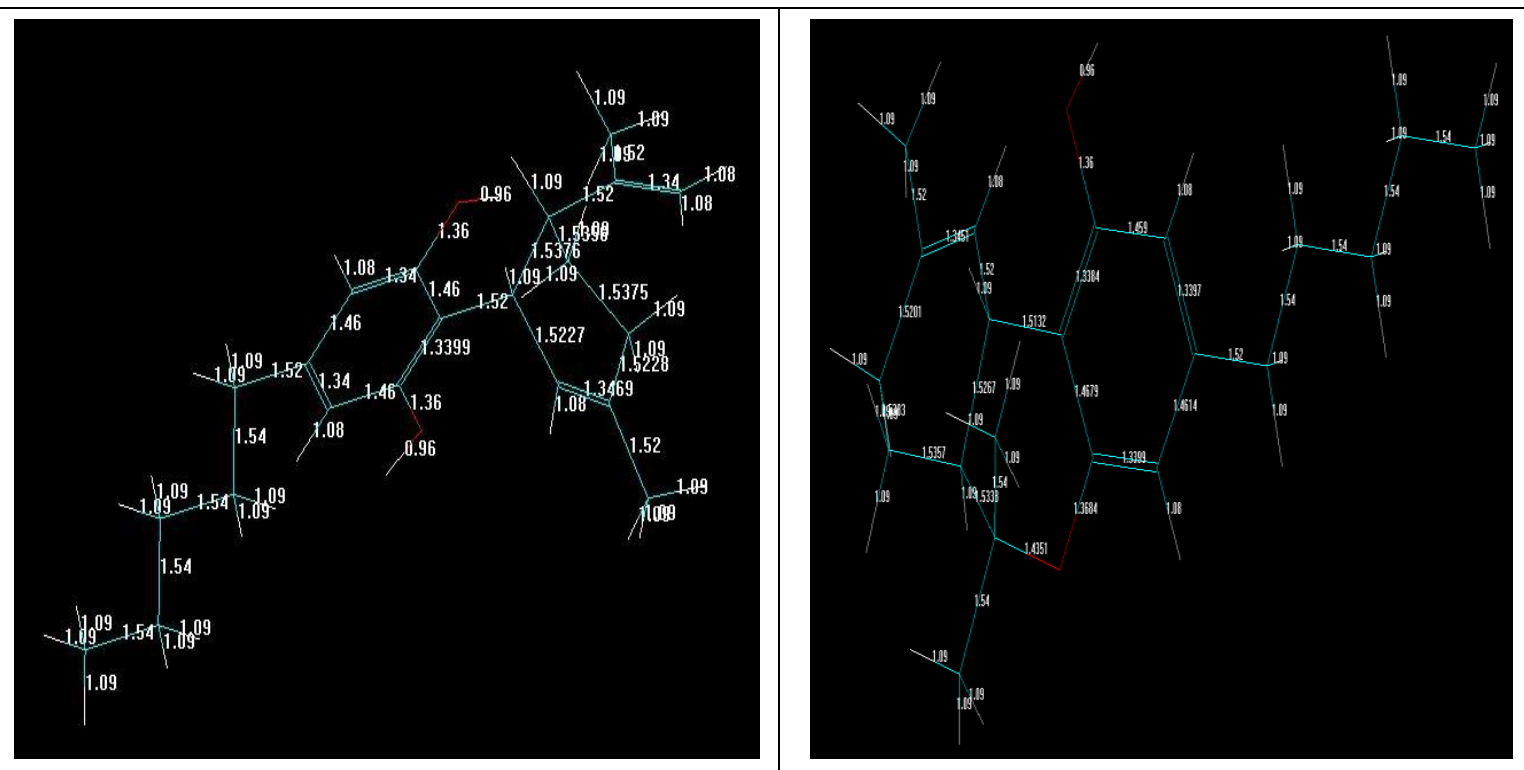

Figure 2. Bond Length of CBD and THC 


\section{Bond order}

The higher the bond order, are considered as the stronger the pull between the two atoms and the shorter the bond length. The shorter bond length indicates the higher required energy as a result the rate of reaction decreases and all other atoms have bond order 1 indicating the strongest pull.

\section{Thermophysical properties}

The binding free energy of the optimized molecules is calculated by performing computing by semiempirical method. The molecule with minimum binding energy will have the maximum binding affinity. The binding free energy of the designed molecules is obtained by eliminating the energy of the main molecule. Having the maximum binding affinity, indicating as the best molecule for drug leads molecules targeting computationally. We can find out the drug binding affinity by using the fitness of the drug, which can bind to the target molecule during the computing process and the second way is using Gibbs free energy calculations. According to this more negative value, we can consider a more effective drug [21].

The bond dipole moment is the idea of electric dipole moment to measure the polarity of a chemical bond within a molecule. In view of pharmaceutical industries, the dipole moment was a parameter for drug by which a drug is to be used open or close packet. If any drug has dipole moment, it can be able to absorb the ultra violet light and have a possibility of properties. The two optimized ILs show zero dipole moment so that it can be used without any protection from UV or sun light.

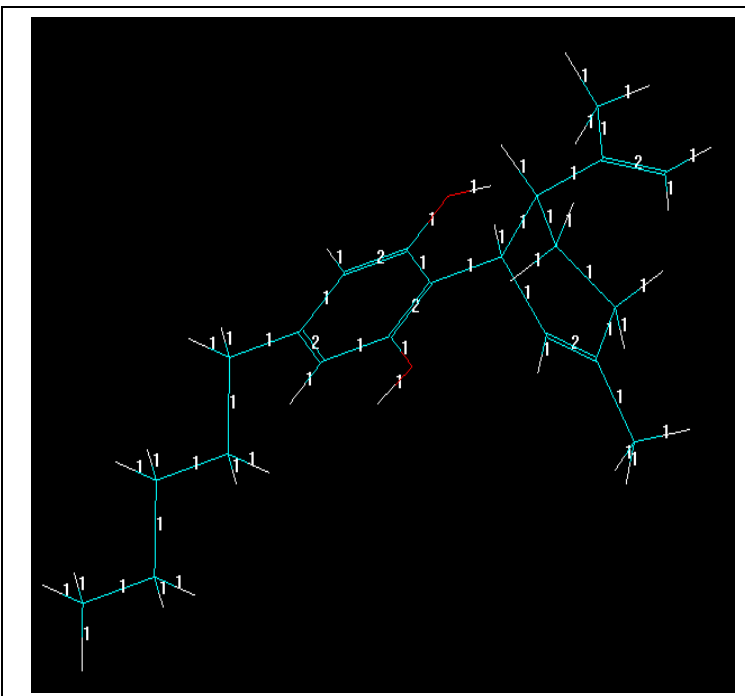

CBD

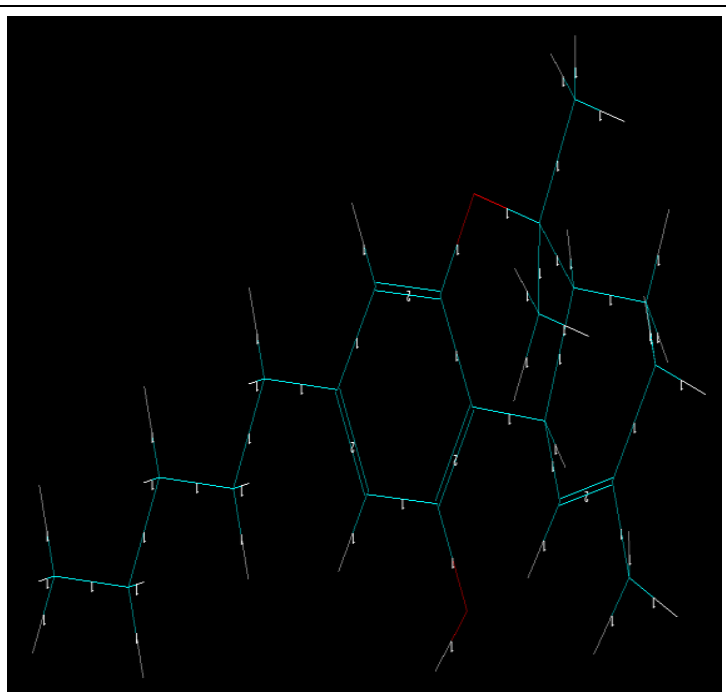

THC

Figure 3. Bond order of CBD and THC 
Table 1. Thermophysical properties

\begin{tabular}{ccc}
\hline Properties & CBD & THC \\
\hline Total energy, (kcal/mol) & 124553.5284 & -81637.91824 \\
Entropy, (kcal/mol-deg) & 0 & 0 \\
Free energy, (kcal/mol) & 124553.5284 & -81637.91824 \\
Heat capacity, (kcal/mol-deg) & 0 & 0 \\
Dipole moment, $(\mathrm{D})$ & 1919 & 0 \\
RMS gradient, $(\mathrm{kcal} / \mathrm{mol})$ & 1584 & 0.4594 \\
Binding energy, $(\mathrm{kcal} / \mathrm{mol})$ & 200809.4294 & -5382.017296 \\
Heat of formation, $(\mathrm{kcal} / \mathrm{mol})$ & 206080.2974 & -111.1492957 \\
Electronic energy, $(\mathrm{kcal} / \mathrm{mol})$ & -477604.9909 & -686792.9782 \\
Nuclear energy, $(\mathrm{kcal} / \mathrm{mol})$ & 602158.5194 & 605155.06 \\
\hline
\end{tabular}

Table 2. HOMO, LUMO

\begin{tabular}{ccccccccc}
\hline \multirow{2}{*}{ CBD } & Levels & $\mathbf{- 3}$ & $\mathbf{- 2}$ & $\mathbf{- 1}$ & $\mathbf{0}$ & $\mathbf{1}$ & $\mathbf{2}$ & $\mathbf{3}$ \\
\cline { 2 - 9 } & HOMO,(eV) & 11.528 & 0.553 & -6.718 & -8.265 & -32.577 & -55.035 & -66.442 \\
& LUMO,(eV) & -55.035 & -32.577 & -8.265 & -6.718 & 0.553 & 11.528 & 12.861 \\
& HOMU- LUMO & +65.563 & +33.130 & -1.547 & -1.547 & -33.130 & -65.563 & -79.303 \\
& gap, (eV) & & & & & & & \\
& HOMO,(eV) & 1.896 & 0.513 & 0.152 & -8.491 & -8.901 & -9.254 & -10.060 \\
& LUMO,(eV), & -9.254 & -8.901 & -8.491 & 0.152 & 0.513 & 1.896 & 1.925 \\
\multirow{2}{*}{ THC } & HOMO- LUMO & +11.150 & +9.414 & +8.643 & -8.643 & -9.44 & -11.150 & 11.985 \\
& gap, $(\mathrm{eV})$ & & & & & & & \\
\hline
\end{tabular}

\section{HOMO-LUMO}

The energy levels of the molecular orbital order HOMO (Highest Occupied Molecular Orbital) and LUMO (Lowest Unoccupied Molecular Orbital) for molecules give information on the possible electronic transition. The HOMO and LUMO also indicate the electrophilic and nucleophilic attraction in molecule. The LUMO-HOMO gap is the most important parameter for the chemical reactivity. The shorter LUMOHUMO gap is considered as the high reactivity, they are highlighted in Figure 2 (color: green is positive value and blue is negative value. The electrophilic (Positive charge groups or atoms) attack to the most likely to the atomic site with a high density of orbital HOMO, while nucleophilic (Negative charge groups or atoms) attack LUMO that is correlated with atomic highdensity of orbital LUMO.

\section{Vibrational spectrum}

The Vibrational spectra of CBD and THC confirmed the presence of $\mathrm{NH}$ from the symmetric and asymmetric stretching peak at $\sim 1770 \mathrm{~cm}-1$ to $\sim 1550 \mathrm{~cm}^{-1}$ respectively, 
with the former being overlapped by $\mathrm{N}-\mathrm{H}$ vibrations. The peak of $-\mathrm{OH}$ is about 3300 to $3500 \mathrm{~cm}^{-1}$.

The distribution electrostatic potential due to 3D mapped structure

The stability of the studied molecular structure is given by the higher negative values of total energy. The biological activity of a compound can be estimated on the basis of the energy difference $\Delta \mathrm{E}$ frontier orbital. This difference, $\Delta \mathrm{E}$ represents the electronic excitation energy which is possible in a molecule. According the mechanism of antimicrobial activity and antimicrobial agents of bioactive molecules, the positive charge end of molecules is responsible to damage the plasma membrane of pathogens [22].

\section{HOMO and LUMO Orbital Diagram of CBD and THC}

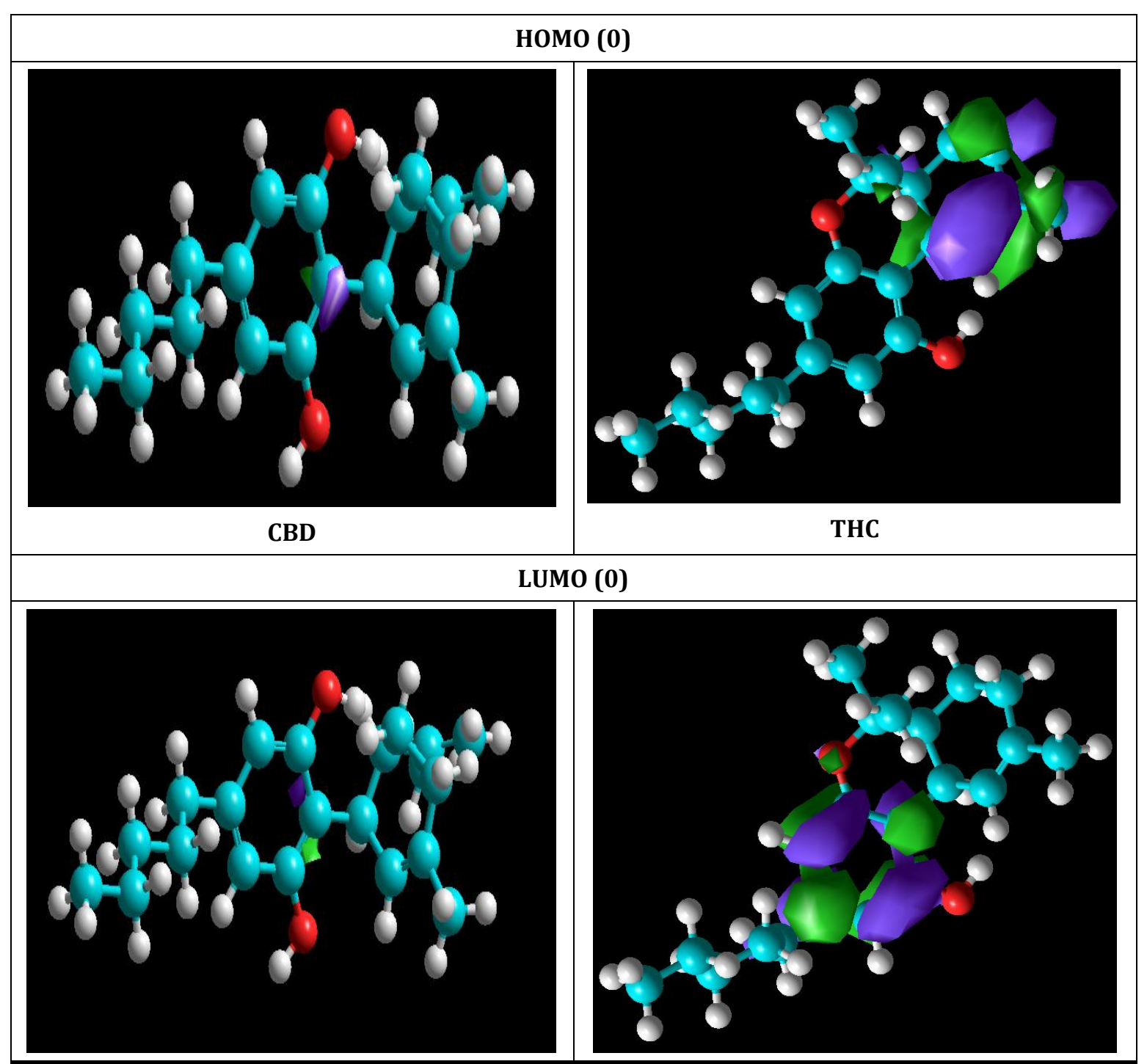

Figure 4. HOMO, LUMO orbital diagram of CBD and THC 


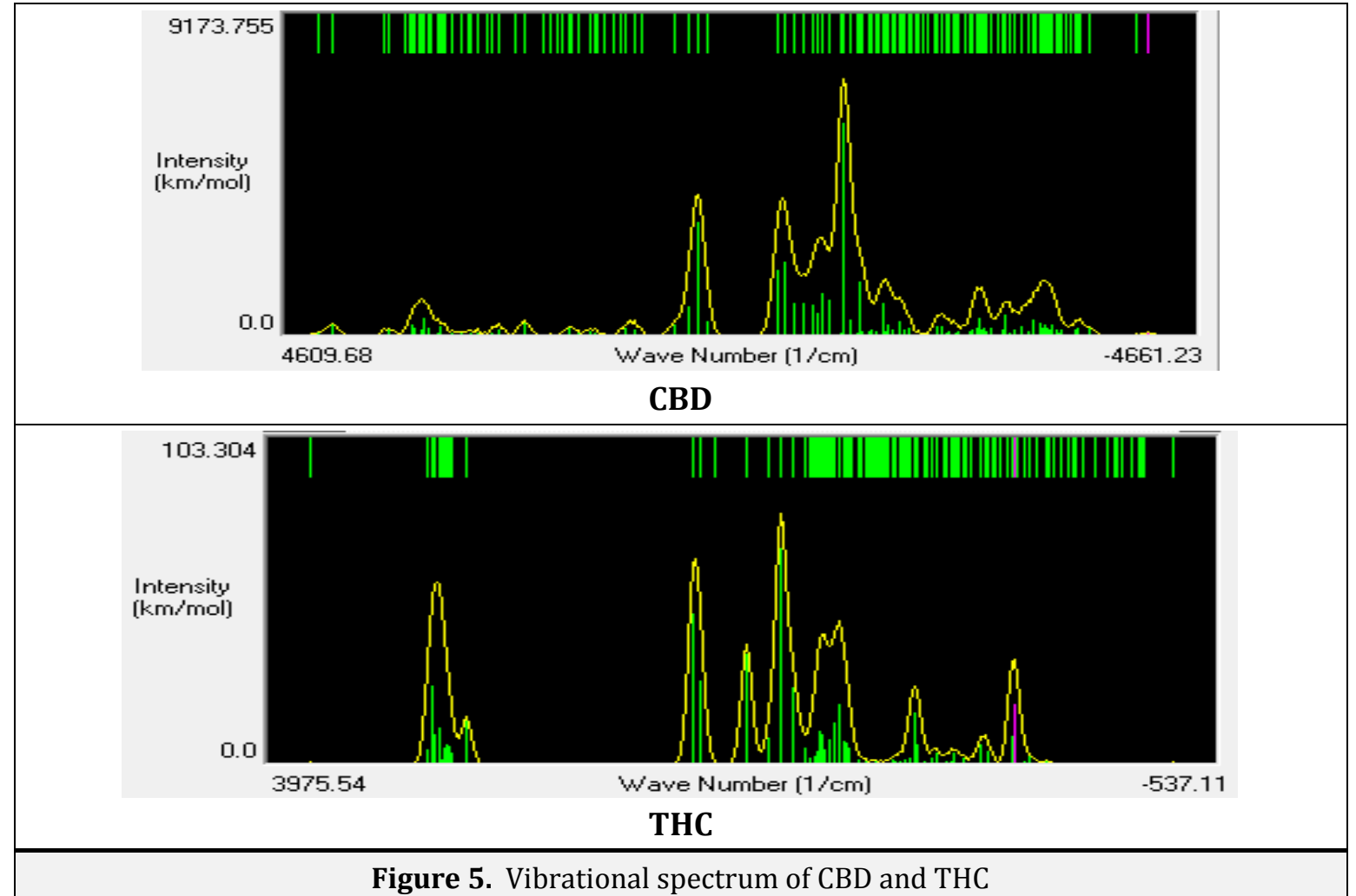

Figure 5. Vibrational spectrum of CBD and THC

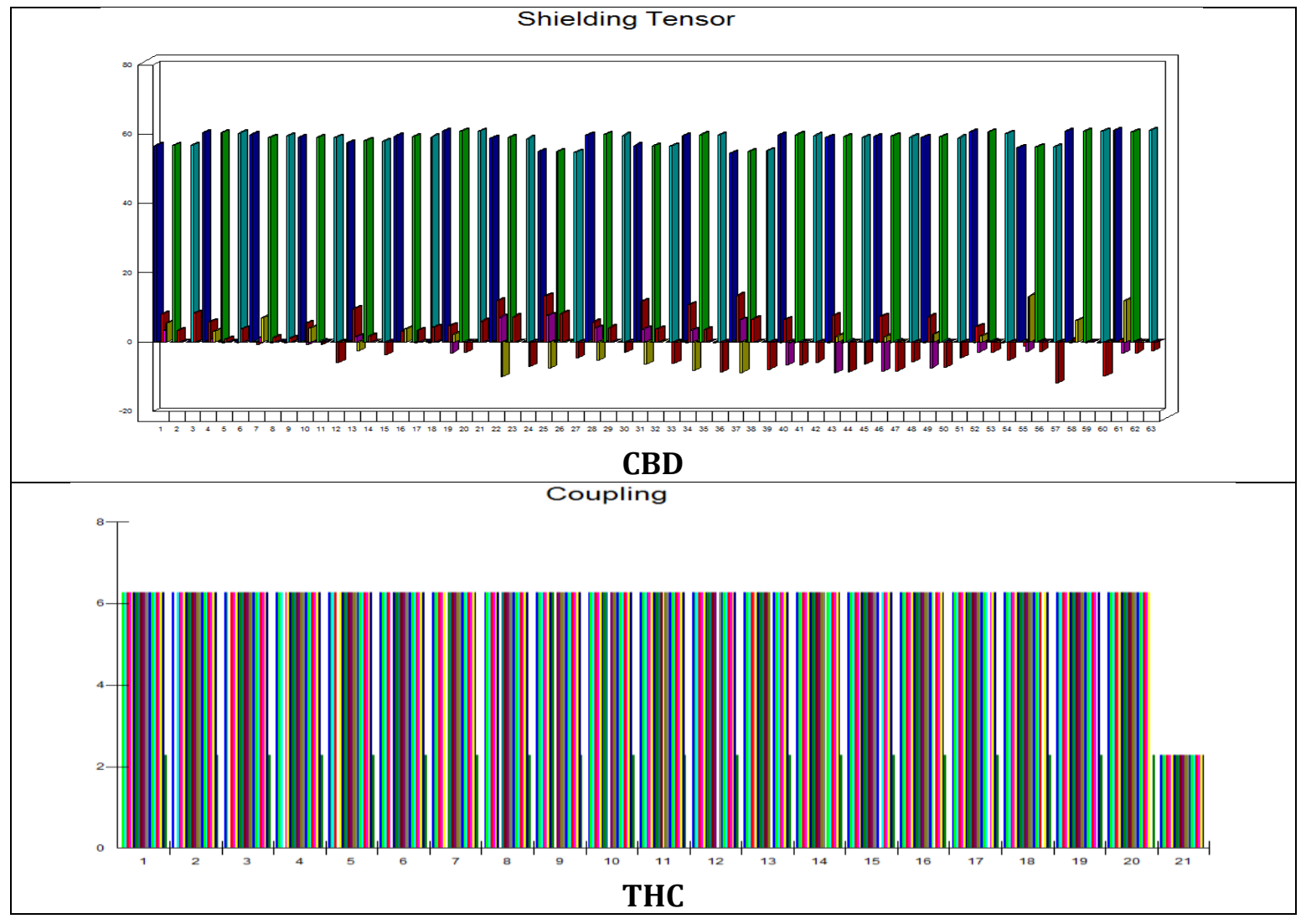

197 Adv J Chem A 2019, 2(3), 190-202 | http://ajchem-a.com 


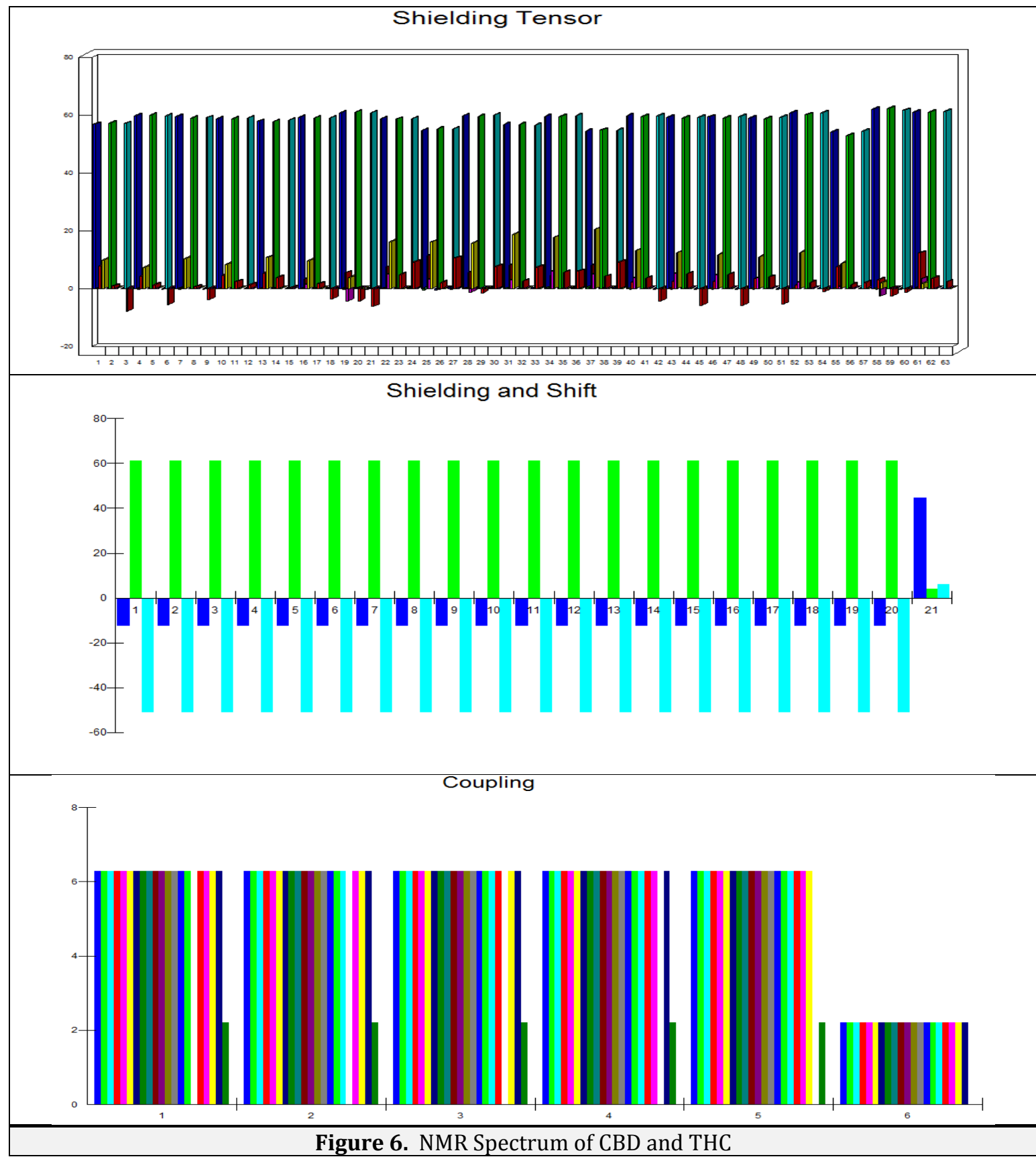

To kill to pathogens, the region of molecules was used the positive charge area of the molecule. In this case the most important factors is explained that the higher surface area having positive charge is considered as the high antimicrobial activity. The electrostatic potential in view of 3D mapped structure indicates positive and negative charge region and charged surface area in a molecule [23] that is considered as the best tools to estimate the biological activity parameters [24]. 


\section{Quantitative Structure Activity Relationship (QSAR) study}

QSAR, a quantum chemical technique is considered as biological activity parameter of compounds which has been deduced by molecular structure of compound and has been extensively used as predicting tool in rational drug design. The QSAR is one of the most important areas in chemometrics and bioinformatics for predicting biological activity. The QSAR models are mathematical equations relating chemical structure to their biological activity. QSAR

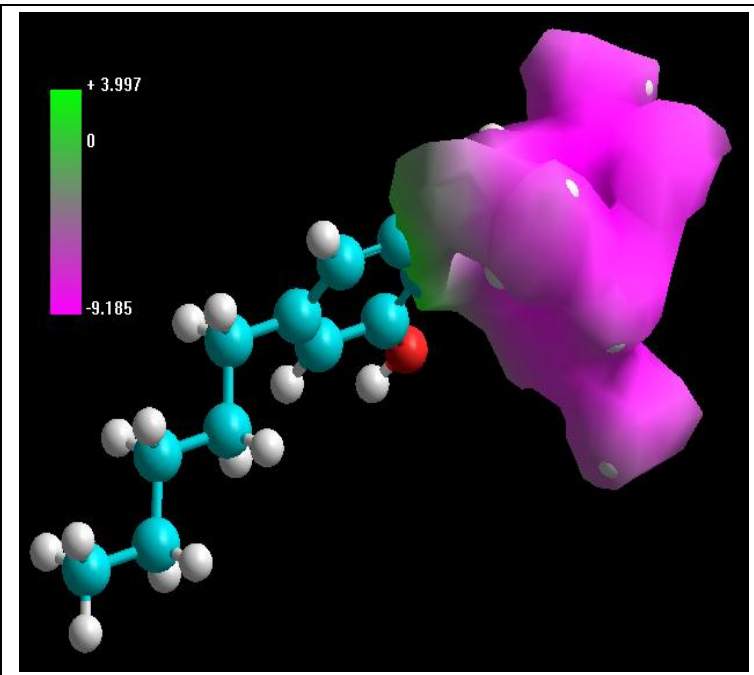

CBD are attempts to correlate molecular structure, or properties derived from molecular structure with a particular kind of chemical or biochemical activity. In case of the biological activity of a molecule, the surface area is considered as the important parameter. Greater charge surface area of a molecule can be able to kill more pathogens. The charged distribution from electrostatic potential completely depends on the surface area. The greater positive charge surface area means the higher biological activity.

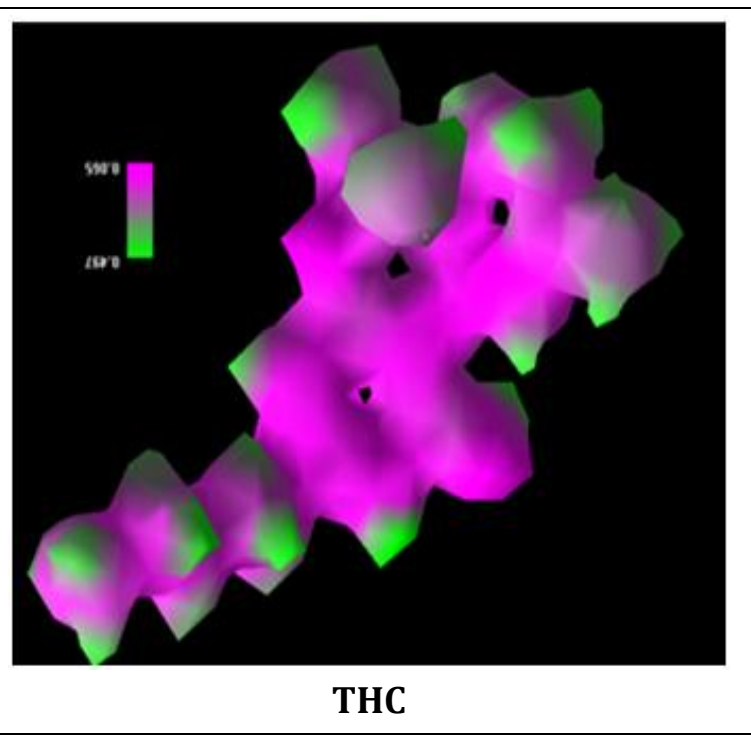

Figure 7. Electrostatic Potential Due to 3D Mapped Structure of CBD and

Table 3. QSAR data

\begin{tabular}{ccc}
\hline & CBD & THC \\
\hline Partial charge (e) & 0 & 0 \\
Surface Area, (grid) & 586.80 & 75.38 \\
Molar Volume, $\AA^{3}$ & 997.96 & 980.22 \\
Hydration Energy, kcal/mol & -3.47 & -0.77 \\
Log P & 3.41 & 2.79 \\
Molar Refractivity $\AA^{3}$ & 101.16 & 99.65 \\
Polarizibility, $\AA^{3}$ & 38.08 & 37.49 \\
Molecular weight, (amu) & 314.47 & 314.47 \\
\hline
\end{tabular}


Table 4. Data od PIC50, IC50

\begin{tabular}{ccc}
\hline & PIC50 & IC50 \\
\hline CBD & 7.436 & $3.664 \times 10^{-08}$ \\
THC & -3.431 & $3.70 \times 10^{-04}$ \\
\hline
\end{tabular}

Table 5. Data of ionization potential (I), electron affinity (A), Hardness, and Softness

\begin{tabular}{cccccc}
\hline & HOMO-LUMO & Hardness $\boldsymbol{\eta}$ & Softness S & $\begin{array}{c}\text { Ionization } \\
\text { potential }\end{array}$ & $\begin{array}{c}\text { Electron } \\
\text { Affinity }\end{array}$ \\
\hline CBD & -1.547 & -0.7735 & 1.2928 & -8.265 & -6.718 \\
THC & -8.643 & 4.3215 & 0.3214 & -8.491 & 0 \\
\hline
\end{tabular}

\section{Calculation of PIC50}

The correlation between the biological activity and descriptor is developed by Zineb Almi et.al. 2014 [25] for the PIC50 value calculation from the Hyperchem simulation value that is given in following equation as:

$$
\begin{aligned}
\text { PIC50 }=3.028 & -0.542 \log \mathrm{P}+0.352 \mathrm{HE}-1.272 \mathrm{Pol}+0.863 \mathrm{MR}-0.038 \mathrm{MV}-0.024 \mathrm{MW} \\
& +19.120 \mathrm{q} 01+0.024 \mathrm{SAG}
\end{aligned}
$$

Here, HE=Hydration Energy, Pol= and LUMO energy values like as IP = Polazibility, $\mathrm{MR}=$ Molecular Refractivity, $\log \mathrm{P}=$ Partition coefficient, $\mathrm{MV}=$ Molar Similar ways, we know that, pIC50 = -log IC50, IC50 $=\log ^{-1}[-$ IC50], Volume, MW= Molar Weight, SAG= Surface Area Grid, q01= atomic net charges.

From the above table, it is shown that, the THC has greater IC50 value that CBD and could be said that it is highly biological active that CBD.

Calculation of ionization potential (I), electron affinity (A), Hardness, and Softness

The ionization potential (I) and electron affinity (A) can be estimated from the HOMO Negative of the energy of HOMO, and EA = Negative of the energy of LUMO.

Hardness $\eta=\frac{\text { (HOMO-LUMO) }}{2}$

Softness $S=\frac{1}{\eta}$

\section{Conclusion}

The most important properties for biological chemistry, chemical reactivity and molecular orbital study like QSAR, HOMO, LUMO, HOMO-LUMO gap, ionization potential, electron affinity, and electrostatic potential in case of the charge distribution in molecule were optimized which indicates that it is good drug molecules. In the case of PIC 50 evaluation, it is shown that the THC is 
more biological active than CBD.

\section{Acknowledgment}

I am thankful to Prof. Dr. Mukbul Ahmed Khan, V.C of European University of Bangladesh and also thankful to Mr Zemam Ahmed Head of IT department and Mr. Moinul Islam, Assistant IT Officer, European University of Bangladesh, for all kind of technical supports.

\section{ORCID}

Ajoy Kumar (D) : 0000-0001-5136-6166

\section{Reference}

[1]. T.H. Moore, S. Zammit, A. LingfordHughes, T.R. Barnes, P.B. Jones, M. Burke, G. Lewis, Lancet, 2007, 370, 319-328.

[2]. M.A. ElSohly, D. Slade, Life Sci., 2005, 78, 539-548.

[3]. V. Auwärter, S. Dresen, W. Weinmann, M. Müller, M. Pütz, N. Ferreirós, J. Mass Spectrom., 2009, 44, 832-837.

[4]. T. Nadulski, F. Pragst, G. Weinberg, P. Roser, M. Schnelle, E.M. Fronk, A.M. Stadelmann, Ther. drug monit., 2005, 27, 799-810.

[5]. I.G. Karniol, I. Shirakawa, N. Kasinski, A. Pfeferman, E.A. Carlini, Eur. J. pharm., 1974, $28,172-177$.

[6]. A.N. Nicholson, C. Turner, B.M. Stone, P.J. Robson, J. clin. Psychopharm., 2004, 24, 305313.

[7]. M. Naef, M. Curatolo, S. Petersen-Felix, L.
Arendt-Nielsen, A. Zbinden, R. Brenneisen, Pain, 2003, 105, 79-88.

[8]. S. Bhattacharyya, P.D. Morrison, P. Fusar-Poli, R. Martin-Santos, S. Borgwardt, T. Winton-Brown, C. Nosarti, C.M. O'Carroll, M. Seal, P. Allen, M.A. Mehta, Neuropsychopharmacology, 2010, 35, 764774.

[9]. R. Martin-Santos, J. a Crippa, A. Batalla, S. Bhattacharyya, Z. Atakan, S. Borgwardt, P. Allen, M. Seal, K. Langohr, M. Farre, A.W. Zuardi, Curr. Pharm. Des., 2012, 18, 49664979.

[10]. M.D. Srivastava, B. Srivastava, B. Brouhard, Immunopharmacology, 1998, 40, 179-185.

[11]. P. Nagarkatti, R. Pandey, S. A. Rieder, V. L. Hegde, M. Nagarkatti, Future Med. Chem., 2009, 1, 1333-1349.

[12]. M. Lodzki, B. Godin, L. Rakou, R. Mechoulam, R. Gallily, E. Touitou, J. Control. Release., 2003, 93, 377-387.

[13]. R.B. Zurier, J. Cell. Biochem., 2003, 88, 462-466.

[14]. D. Sulak, R. Saneto, B. Goldstein, Epilepsy Behav., 2017, 70, 328-333.

[15]. E. National Academies of Sciences, Medicine, The health effects of cannabis and cannabinoids: The current state of evidence and recommendations for research. Editor, National Academies Press, 2017.

[16]. S. Steffens, N.R. Veillard, C. Arnaud, G. Pelli, F. Burger, C. Staub, M. Karsak, A. 
Zimmer, J.L. Frossard, F. Mach, Nature, 2005, 434, 782-786.

[17]. B. Costa, A.E. Trovato, F. Comelli, G. Giagnoni, M. Colleoni, Eur. J. Pharm., 2007, $556,75-83$.

[18]. M.W. Elmes, M. Kaczocha, W.T. Berger, K. Leung, B.P. Ralph, L. Wang, J.M. Sweeney, J.T. Miyauchi, S.E. Tsirka, I. Ojima, D.G. Deutsch, J. Biol. Chem., 2015, 290, 87118721.

[19]. A. Howard, J. McIver, J. Collins, Hyperchem computational chemistry. Hypercube Inc., Waterloo, 1994.
[20]. R.S, Mulliken, J. Chem. Phys., 1955, 23, 1841-1846.

[21]. A.R. Ortiz, M.T. Pisabarro, F. Gago, R.C. Wade, J. Med. Chem., 1995, 38, 2681-2691. [22]. L. Timofeeva, N. Kleshcheva, Appl. Microbial. Biotechnol., 2011, 89, 475-492.

[23]. K.D. Collins, Biophys. J., 1997, 72, 65-76. [24]. M. Böhm, J. Stürzebecher, G. Klebe, J. Med. Chem., 1999, 42, 458-477.

[25]. S.B. Zineb Almi, T. Lanez, N. Tchouar, Int. Lett. Chem. Phys. Astron., 2014, 37, 113124.

How to cite this manuscript: Ajoy Kumer, Nuruzzaman Sarkar, Sunanda Paul, Afroza Zannat, The Theoretical Prediction of Thermophysical properties, HOMO, LUMO, QSAR and Biological Indics of Cannabinoids (CBD) and Tetrahhdrocannabinol (THC) by Computational Chemistry, Advanced Journal of Chemistry-Section A (Adv. J. Chem. A), 2019, 2(3), 190-202. 